\title{
Development of Cassette PAMPA for Permeability Screening
}

\author{
Mun Hwan Oh, ${ }^{a}$ Hye Jin Lee, ${ }^{a}$ Sang Hyun Jo, ${ }^{a}$ Bernie Byunghoon Park, ${ }^{a}$ Sae-Bom Park, ${ }^{a}$ \\ Eun-yeong Kim, ${ }^{a}$ Yuanyuan Zhou, ${ }^{a}$ Young Ho Jeon, ${ }^{a}$ and Kiho Lee*,a,b \\ ${ }^{a}$ College of Pharmacy, Korea University; Sejong 30019, Republic of Korea: and ${ }^{b}$ Biomedical Research Center, Korea \\ University Guro Hospital; Seoul 08308, Republic of Korea.
}

Received September 21, 2016; accepted January 26, 2017

\begin{abstract}
The parallel artificial membrane permeability assay (PAMPA) is widely used in early-stage drug discovery to discriminate compounds by intestinal permeability. The purpose of the current study was to establish a cassette (n-in-1) PAMPA to enable permeability screening of lipophilic compounds. A double-sink PAMPA consisting of a pH gradient (i.e., pH 6.5 and 7.4 for the donor and receiver compartments, respectively) and a lipophilic sink (i.e., a surfactant in the receiver solution) was utilized with cassette incubation of 10 reference compounds. Sample analysis was conducted using selected reaction monitoring (SRM) with a triple quadrupole LC-MS/MS system. Correlation between PAMPA permeability and human intestinal absorption (HIA) of the reference compounds yielded two false negatives, namely propranolol (PPN) and verapamil (VER); these two compounds showed a substantially lower recovery (ca. 10\%) than other reference compounds ( $>69 \%$ ). This cassette PAMPA was repeated subsequently with polysorbate 80 added to the donor compartments, which resulted in a significant increase in both the recovery and the permeability of the false negatives. Accordingly, the permeability class of all reference compounds could be unambiguously differentiated using this cassette PAMPA. Also, a strong linear correlation $(r=0.9845)$ was observed between the cassette and discrete permeability of all reference compounds.
\end{abstract}

Key words parallel artificial membrane permeability assay (PAMPA); cassette; permeability; nonspecific binding; lipophilic compound

The in vitro assays used to evaluate the intestinal permeability of new chemical entities (NCEs) are classified into cellular and non-cellular methods. ${ }^{1)}$ The Caco-2 and MadinDarby canine kidney (MDCK) cell permeability assays are representative of the cellular methods. ${ }^{2)}$ Two representative non-cellular methods are the parallel artificial membrane permeability assay (PAMPA) and the phospholipid vesicle-based permeation assay (PVPA). ${ }^{1)}$ PAMPA is more cost-effective, less complex, and less time-consuming than the cellular methods. PAMPA therefore has been widely used, especially in early drug discovery, to discriminate lead compounds by human intestinal permeability, ${ }^{1)}$ despite the limitation that it can only test for passive diffusion across lipid membranes (i.e., transcellular drug absorption).

Lipophilic compounds constitute a significant portion of the compounds generated in the early stages of recent drug discovery processes. ${ }^{3-5)}$ Lipophilic compounds in general have low solubility in the buffers used in absorption, distribution, metabolism, and excretion (ADME) assays commonly implemented during drug discovery, including PAMPA. ${ }^{1)}$ Various solubilizing agents, including co-solvents and non-ionic surfactants such as Brij 35, polysorbate 80, and Cremophor EL, have been used to increase compound solubility in PAMPA. ${ }^{1}$ In addition, nonspecific binding such as adsorption of lipophilic compounds to the plastic surface and filter support and accumulation within the lipid membrane could result in poor recovery (poor mass balance) and an underestimation of permeability in the PAMPA assay ${ }^{1)}$; this problem could also be alleviated by using solubilizing agents.

Cassette incubation followed by LC-MS analysis using an Orbitrap high resolution mass spectrometer or a single quadrupole mass spectrometer has been implemented in PAMPA in an effort to increase throughput. ${ }^{6,7)}$ In the current study we establish a cassette PAMPA assay that would be especially useful for screening the permeability of lipophilic compounds. A double-sink PAMPA consisting of a $\mathrm{pH}$ gradient (i.e., $\mathrm{pH}$ 6.5 and 7.4 for the donor and receiver compartments, respectively) and a lipophilic sink (i.e., a surfactant in the receiver solution) was utilized with cassette incubation of reference compounds in our studies. ${ }^{8}$ Sample analysis was performed using selected reaction monitoring (SRM) with a triple quadrupole LC-MS/MS system. This sensitive and selective bioanalytical technique capable of simultaneous quantitation of all test compounds will allow for permeability screening of poorly soluble, lipophilic compounds that should be tested at low concentrations.

\section{MATERIALS AND METHODS}

Materials Dimethyl sulfoxide (DMSO), $n$-dodecane, soybean L- $\alpha$-phosphatidylcholine (PC), formic acid, potassium phosphate monobasic dihydrate, potassium phosphate dibasic, polysorbate 80 , calcein, Lucifer Yellow $\mathrm{CH}$ dipotassium salt, and D- $\alpha$-tocopheryl polyethylene glycol 1000 succinate (TPGS) were bought from Sigma-Aldrich Korea (Yongin, Kyeonggi, Republic of Korea). The reference compounds, atenolol (ATN), nadolol (NDL), norfloxacin (NFX), ranitidine hydrochloride (RTD), verapamil hydrochloride (VER), chloramphenicol (CLP), hydrochlorothiazide (HCT), and ketoprofen (KTP) and the analytical internal standards (IS) carbamazepine (CMP) and glipizide (GPZ) were also purchased from Sigma-Aldrich Korea. The reference compounds metoprolol (MTP) and propranolol (PPN) were gifts from the Bio-evaluation Center, KRIBB (Cheongju, Chungbuk, Republic of Korea). Millipore Multiscreen 96-well microtiter plates (Cat. No. MATRNPS50) and Millipore Multiscreen-IP 96-well 
microfilter plates (Cat. No. MAIPNTR10) were purchased from Fisher Scientific (Seoul, Republic of Korea) and used as donor and receiver plates for PAMPA assays, respectively. HPLC-grade water and HPLC-grade acetonitrile were purchased from Avantor Performance Materials (Center Valley, PA, U.S.A.).

PAMPA PAMPA was conducted as described previously ${ }^{9)}$ with minor modifications. PC solution was prepared daily at $2 \%(\mathrm{w} / \mathrm{v})$ in $n$-dodecane. $0.1 \mathrm{M}$ phosphate buffer ( $\mathrm{pH} 7.4)$ containing $0.2 \%(\mathrm{w} / \mathrm{v})$ TPGS and 5\% (vol) DMSO was used as the receiver buffer. Donor solutions for cassette PAMPA were prepared by mixing a DMSO stock solution containing all 10 reference compounds at $0.1 \mathrm{~mm}$ each with 19 volumes of $0.1 \mathrm{M}$ phosphate buffer ( $\mathrm{pH}$ 6.5) to give a final compound concentration of $5 \mu \mathrm{M}$ each and a DMSO concentration of $5 \%$ (vol); donor solutions for discrete PAMPA assay were prepared in the same way except that $0.1 \mathrm{~mm}$ DMSO stock solutions of each reference compound were used individually. To test the effect of polysorbate 80 on permeability and nonspecific binding in PAMPA, donor solutions were prepared in $0.1 \mathrm{M}$ phosphate buffer ( $\mathrm{pH} 6.5$ ) containing $0.1-1 \%$ (vol) polysorbate 80 in the same way as described above. PAMPA was initiated by adding $300 \mu \mathrm{L}$ of donor solution to each well of the donor plate. The filter of each receiver well was impregnated with $6 \mu \mathrm{L}$ of the $2 \%(\mathrm{w} / \mathrm{v})$ PC solution followed immediately by addition of $150 \mu \mathrm{L}$ of the receiver buffer to each receiver well. The receiver plate was then placed onto the donor plate. The assembled assay plates were incubated at $25^{\circ} \mathrm{C}$ for $16 \mathrm{~h}$ under constant orbital shaking at $300 \mathrm{rpm}$. The plates were then separated, and $100 \mu \mathrm{L}$ aliquots from each well of both plates were transferred to a 96 -well plate containing $100 \mu \mathrm{L}$ of the opposite matrix (i.e., receiver buffer for donor samples and donor buffer for receiver samples) for matrix matching. One hundred microliters of dosing solution was mixed with the same volume of the receiver buffer to prepare a time 0 donor sample. Finally, $100 \mu \mathrm{L}$ of acetonitrile containing both CMP (IS for positive ionization mode) and GPZ (IS for negative ionization mode) was added to each well. The prepared samples were analyzed with a triple quadrupole LC-MS/MS system in multiple reaction monitoring (MRM) mode as described below.

LC-MS/MS Analytical Method An Agilent 6460 triple quadrupole LC-MS/MS system equipped with a dual AJS ESI ion source and an Agilent 1200 series HPLC system was used. Chromatographic separation was performed on an Agilent Eclipse plus $\mathrm{C} 18$ column $(2.1 \times 100 \mathrm{~mm}, 4.6 \mu \mathrm{m})$ with $0.1 \%$ (vol) formic acid in deionized water (mobile phase A) and $0.1 \%$ (vol) formic acid in acetonitrile (mobile phase B) run in the following elution gradient: $2 \% \mathrm{~B}$ for the initial $2 \mathrm{~min}$, linear gradient to $35 \% \mathrm{~B}$ for $2 \mathrm{~min}$, and linear gradient to $98 \% \mathrm{~B}$ for $10 \mathrm{~min}$. The flow rate was $0.65 \mathrm{~mL} / \mathrm{min}$. The column temperature was set at $40^{\circ} \mathrm{C}$ and the injection volume was $5 \mu \mathrm{L}$. The ion source parameters of the mass spectrometer include gas temperature of $300^{\circ} \mathrm{C}$, gas flow of $5 \mathrm{~L} / \mathrm{min}$, nebulizer gas pressure of $45 \mathrm{psi}$, sheath gas temperature of $250^{\circ} \mathrm{C}$, and sheath gas flow of $6 \mathrm{~L} / \mathrm{min}$ in both positive and negative SRM modes. The dwell time was $30 \mathrm{~ms}$ for each SRM transition. Other mass spectrometer conditions for each compound such as fragmentor voltage (F), collision energy (CE), $\mathrm{m} / \mathrm{z}$ values of precursor and product ions, and ionization mode are shown in Table 1.

Assessment of Membrane Integrity A membrane integrity test was conducted in the same way as the PAMPA assay described above using calcein and Lucifer Yellow $\mathrm{CH}$ as hydrophilic fluorescent markers. ${ }^{10-12)}$ Donor solutions were prepared with calcein and Lucifer Yellow $\mathrm{CH}$ at a final concentration of $500 \mu \mathrm{M}$ in $0.1 \mathrm{M}$ phosphate buffer ( $\mathrm{pH}$ 6.5) containing $0-3 \%$ (vol) polysorbate 80 . The fluorescence of the receiver solutions was measured after $16 \mathrm{~h}$ incubation at $25^{\circ} \mathrm{C}$ with a Gemini EM Microplate Reader (Molecular Devices, Sunnyvale, CA, U.S.A.); the respective excitation and emission wavelengths were 445 and $512 \mathrm{~nm}$ for calcein, and 435 and $485 \mathrm{~nm}$ for Lucifer Yellow $\mathrm{CH}$.

Calculations Apparent permeability coefficient $\left(P_{\text {app }}\right)$ values were calculated as described by Fortuna et al. ${ }^{9)}$ Sample recovery was calculated using the following equation:

$$
\text { Recovery }(\%)=\frac{\operatorname{Area}_{\mathrm{d}}(t) \cdot V_{\mathrm{d}}+\operatorname{Area}_{\mathrm{r}}(t) \cdot V_{\mathrm{r}}}{\operatorname{Area}_{\mathrm{d}}(0) \cdot V_{\mathrm{d}}} \times 100
$$

where $V_{\mathrm{d}}$ is the volume of donor solution $(0.3 \mathrm{~mL}), V_{\mathrm{r}}$ is the volume of receiver solution $(0.15 \mathrm{~mL}), \operatorname{Area}_{\mathrm{d}}(t)$ is the peak area of the sample from the donor plate at the end of assay, $\operatorname{Area}_{\mathrm{r}}(t)$ is the peak area of the sample from the receiver plate at the end of assay, and Area $_{\mathrm{d}}(0)$ is the peak area of the time 0 donor sample (i.e., dosing solution). Statistical analyses were performed using GraphPad Prism (version 6.0, GraphPad Software, La Jolla, CA, U.S.A.). All statistical tests are one-way

Table 1. LC-MS/MS Analytical Conditions for the Reference Compounds

\begin{tabular}{|c|c|c|c|c|c|c|c|}
\hline \multirow{2}{*}{ Compound } & \multirow{2}{*}{ Nominal mass } & \multicolumn{2}{|c|}{$\operatorname{SRM}(m / z)$} & \multirow{2}{*}{$\mathrm{F}(\mathrm{V})$} & \multirow{2}{*}{$\mathrm{CE}$} & \multirow{2}{*}{$\begin{array}{l}\text { Retention time } \\
\text { (min) }\end{array}$} & \multirow{2}{*}{ Ionization mode } \\
\hline & & Precursor & Product & & & & \\
\hline ATN & 266 & 267 & 145 & 130 & 17 & 3.84 & $(+)$ \\
\hline MTP & 267 & 268 & 56 & 130 & 25 & 4.40 & $(+)$ \\
\hline NDL & 309 & 310 & 254 & 130 & 9 & 4.08 & $(+)$ \\
\hline NFX & 319 & 320 & 187 & 130 & 37 & 4.15 & $(+)$ \\
\hline PPN & 259 & 260 & 56 & 130 & 21 & 4.84 & $(+)$ \\
\hline RTD & 314 & 315 & 102 & 90 & 33 & 3.88 & $(+)$ \\
\hline VER & 454 & 455 & 165 & 170 & 25 & 5.59 & $(+)$ \\
\hline CLP & 322 & 321 & 121 & 90 & 29 & 5.50 & $(-)$ \\
\hline HCT & 297 & 296 & 269 & 170 & 13 & 4.47 & $(-)$ \\
\hline KTP & 254 & 253 & 209 & 90 & 5 & 7.44 & $(-)$ \\
\hline CMP & 236 & 237 & 194 & 135 & 18 & 6.26 & $(+)$ \\
\hline GPZ & 445 & 444 & 319 & 130 & 20 & 6.94 & $(-)$ \\
\hline
\end{tabular}


ANOVAs with Bonferroni post hoc tests; significance between groups is represented as $* p<0.05$, $* * p<0.01$, or $* * * p<0.001$.

\section{RESULTS}

Correlation between Cassette PAMPA Permeability and Human Intestinal Absorption (HIA) PAMPA permeability $\left(P_{\text {app}}\right)$ was first determined for 10 reference compounds (five with HIA $<90 \%$ and five with HIA $\geq 90 \%$ ) added to the donor compartment as a mixture without using any additives in the donor compartment. The HIA (a.k.a. Fa) values were taken from literature. ${ }^{9)}$ All the reference compounds with HIA $<90 \%$, which has been commonly used as a cutoff for low intestinal permeability, ${ }^{9,13)}$ had $P_{\text {app }}$ values $<5 \mathrm{~nm} / \mathrm{s}$ (Fig. 1). Two of the 5 reference compounds with HIA $\geq 90 \%$ also

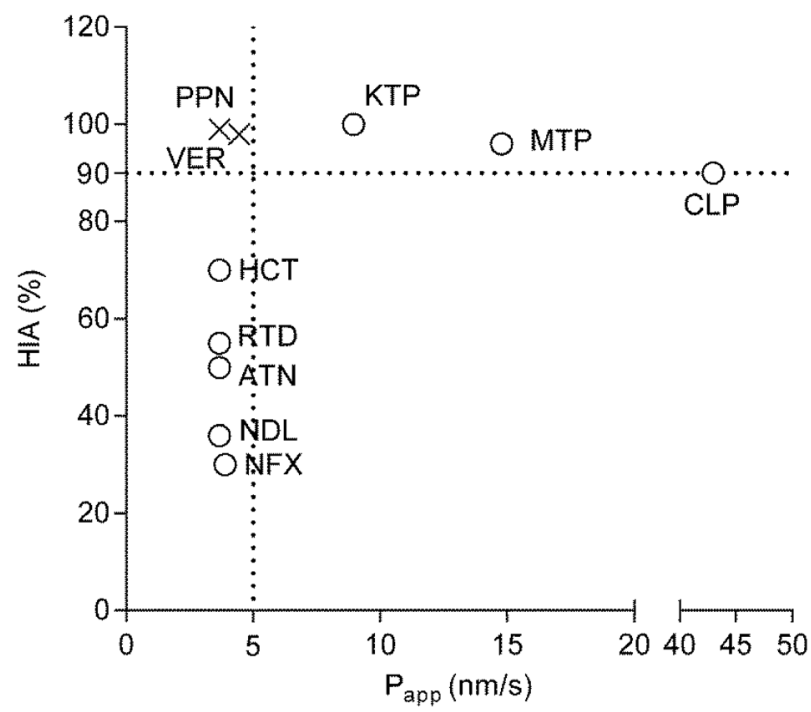

Fig. 1. Correlation between Cassette PAMPA Permeability and HIA

PAMPA permeability $\left(P_{\text {app }}\right)$ was determined for 10 reference compounds added to the donor compartment as a mixture without using any additives in the donor compartment. The concentrations of ATN, HCT, NDL, PPN, and RTD in the receiver compartments were all below the quantitation limit $(i . e .<0.2 \mu \mathrm{M})$, corresponding to a $P_{\text {app }}$ value of $<3.7 \mathrm{~nm} / \mathrm{s}$; the $P_{\text {app }}$ values of these compounds are thus shown as $3.7 \mathrm{~nm} / \mathrm{s}$. Two false negatives, i.e., compounds with low permeability $\left(P_{\text {app }}<5 \mathrm{~nm} / \mathrm{s}\right)$ and high HIA $(\geq 90 \%)$, are marked $(\mathrm{x})$. Data are the mean of 9 measurements.

A

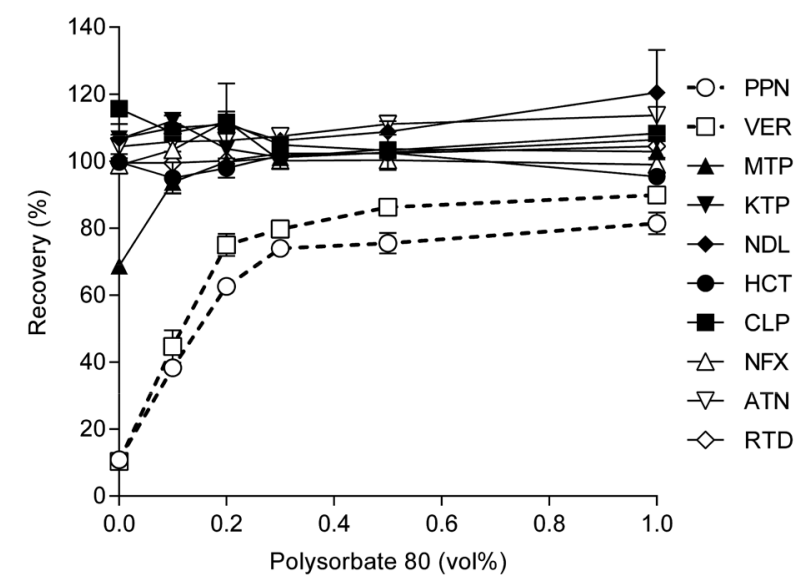

had $P_{\text {app }}$ values $<5 \mathrm{~nm} / \mathrm{s}$ (Fig. 1); these two compounds, PPN and VER, are classified as false negatives when the criteria of $P_{\text {app }}>5 \mathrm{~nm} / \mathrm{s}$ and HIA $\geq 90 \%$ are used for differentiation of high intestinal permeability under these experimental conditions.

Effect of Polysorbate 80 on Recovery and Permeability in Cassette PAMPA The cassette PAMPA described above was repeated in the presence of polysorbate 80 in the donor compartments to test the hypothesis that the permeability of the false negatives had been underestimated due to significant nonspecific binding in the assay system, i.e., to the surface of the assay plate and the lipid membrane. ${ }^{1)}$ Both false negatives had substantially lower recovery ( $c a$. 10\%) than other compounds $(>69 \%)$ when PAMPA was conducted in the absence of polysorbate 80 (Fig. 2A); the 3 compounds, including the two false negatives, showing incomplete recovery had higher $\log P$ values $(\geq 2.00)$ than the ones with complete recovery $(\log P \leq 1.03)$ (Fig. 3). Polysorbate 80 added to the donor compartments increased the recovery of the false negatives up to $>80 \%$ in a concentration-dependent manner (Fig. 2A). The addition of polysorbate 80 also caused a concentrationdependent increase in the permeability of the two false negatives and MTP (Fig. 2B). The recovery and permeability of all other reference compounds that showed low permeability $\left(P_{\text {app }}<5 \mathrm{~nm} / \mathrm{s}\right)$ in the absence of polysorbate 80 , i.e., true negatives, were not affected by the presence of polysorbate 80 in the donor compartments (Figs. 2A, B). These results indicate that the permeability of the two false negatives was likely underestimated because of significant nonspecific binding in the donor compartments. Polysorbate 80 caused a concentrationdependent decrease in the permeability of CLP, although its $P_{\text {app }}$ value remained the highest among all the reference compounds even at the highest concentration of polysorbate 80 (i.e., 1.0\% (vol)) used in this study (Fig. 2B).

Effect of Polysorbate 80 on Membrane Integrity in PAMPA Calcein and Lucifer Yellow $\mathrm{CH}$ were used as hydrophilic fluorescent markers to test the effect of polysorbate 80 on membrane integrity in PAMPA. Polysorbate 80 added to the donor compartments had no significant effect up to $2 \%$ (vol) ( $p>0.05$ by one-way ANOVA) on the fluorescence intensity in the receiver side following a $16 \mathrm{~h}$ incubation with either

B

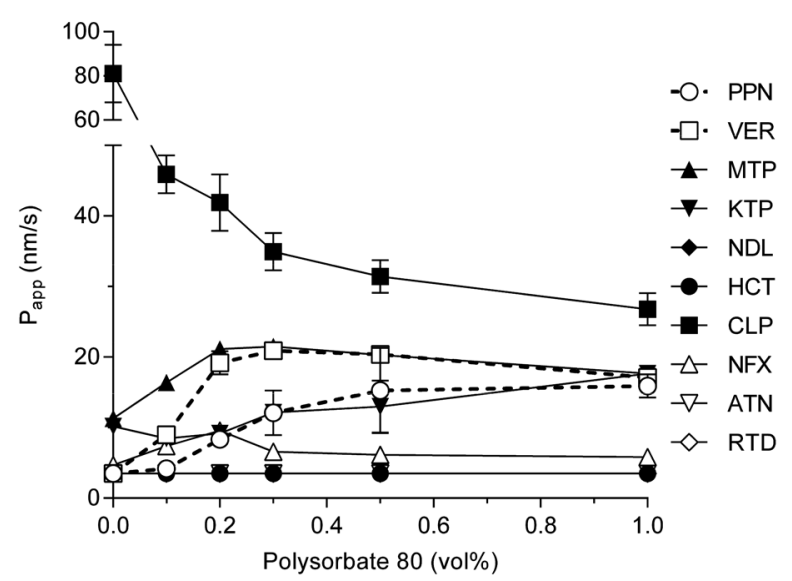

Fig. 2. Effect of Polysorbate 80 on the Recovery (A) and Permeability (B) in Cassette PAMPA

The permeability assay was conducted in cassette mode as described in Fig. 1 in the absence or presence of polysorbate 80 in the donor compartment. Recovery represents $\%$ of each compound recovered from both the donor and the receiver compartments at the end of the assay. Data are mean \pm S.D. $(n=3)$. 


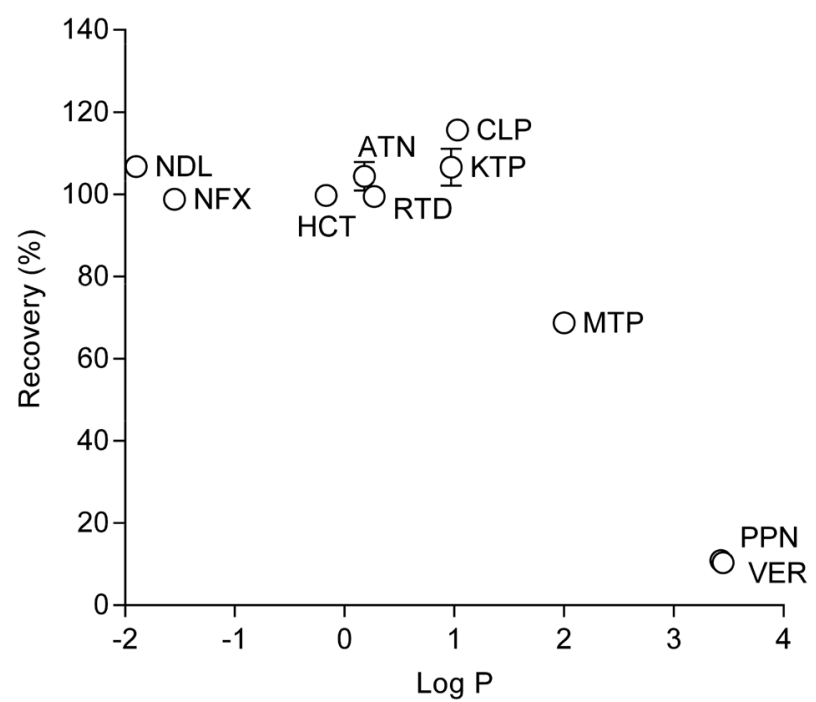

Fig. 3. Correlation between $\log P$ and Recovery of Reference Compounds in Cassette PAMPA

$\log P$ values were taken from the literature (Fortuna et al. 2012). Recovery values were determined as described in Fig. 2. Data are mean \pm S.D. $(n=3)$.

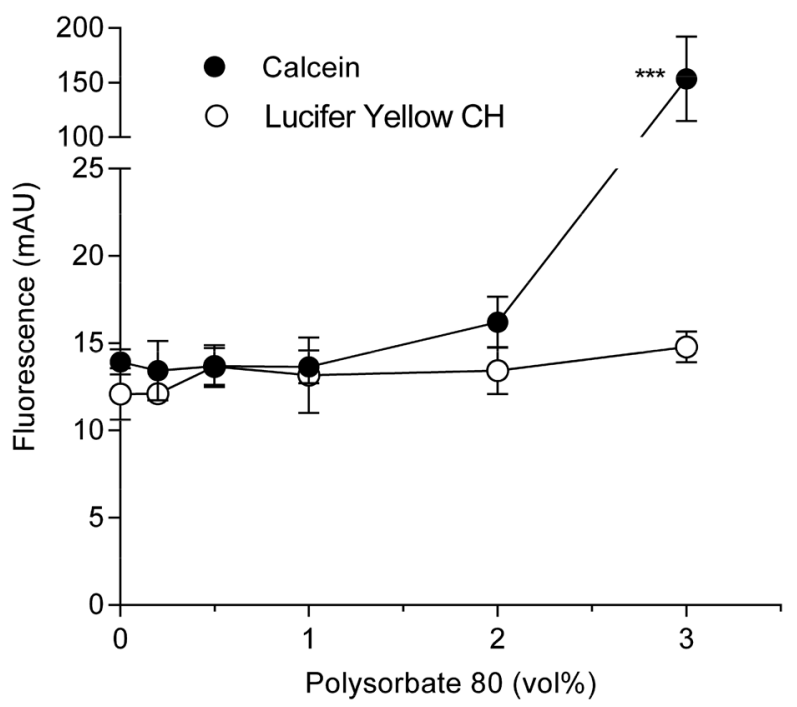

Fig. 4. Effect of Polysorbate 80 on Membrane Integrity in PAMPA

Fluorescence intensity of the receiver solution was measured after a $16 \mathrm{~h}$ incubation with calcein or Lucifer Yellow $\mathrm{CH}$ added to the donor compartments at a fina concentration of $500 \mu \mathrm{m}$ in the presence or absence of polysorbate 80 in the receiver compartments. Data are mean \pm S.D. $(n=3)$. $* * * p<0.001$ (one-way ANOVA).

calcein or Lucifer Yellow $\mathrm{CH}$ in the donor compartments (Fig. 4). This result suggests that membrane integrity was not significantly influenced by polysorbate 80 in this concentration range.

Correlation between PAMPA Permeability Determined in the Presence of Polysorbate 80 and HIA The PAMPA permeability of the 10 reference compounds was determined either individually or in cassette mode with $0.3 \%$ (vol) polysorbate 80 present in the donor compartments. As shown in Fig. 5, the high and low permeability compounds could be unambiguously discriminated from each other in both the cassette (Fig. 5A) and discrete (Fig. 5B) modes when a $P_{\text {app }}$ value of $8 \mathrm{~nm} / \mathrm{s}$ was used as the cutoff. Also, a strong linear relationship was observed between cassette and discrete permeability with a slope close to unity and a Pearson's $r$ value of 0.9845 $(p<0.001)$ (Fig. 6).

\section{DISCUSSION}

With the aim of developing a cassette PAMPA, we first determined the permeability of 10 reference compounds added as a mixture to the donor compartment using a recently published method.9) TPGS was used in the receiver solution in addition to a $\mathrm{pH}$ gradient (i.e., $\mathrm{pH} 6.5$ and 7.4 in the donor and receiver compartments, respectively) to maintain a doublesink condition in our PAMPA assay. ${ }^{14)}$ Among the 10 reference compounds used in this study, the intestinal permeability of only PPN and VER were misclassified when no additives were added to the donor compartment. We later found that these two compounds had a poor recovery of $c a .10 \%$ at the end of the assay, which was much lower than that of other reference compounds. Furthermore, comparison of the $\log P$ values of the reference compounds ${ }^{9)}$ revealed that PPN and VER were the most lipophilic compounds, with $\log P$ values of 3.43 and 3.45 , respectively; the $\log P$ values of other compounds ranged from -1.90 (NDL) to 2.00 (MTP). Subsequent comparison of $\log P$ values of the reference compounds with their \% recovery values led to the finding that there is a roughly inverse relationship between the two variables, suggesting that the poor recovery of PPN and VER was likely caused by nonspecific binding in the assay system.

Polysorbate 80 , a nonionic surfactant, was added to the donor compartment to prevent nonspecific binding and thereby improve the recovery of the test compounds; polysorbate 80 was added only to the donor compartment because another surfactant, TPGS, was already present in the receiver compartment. Polysorbate 80 significantly enhanced the recovery of the 3 reference compounds with incomplete recovery, namely PPN, VER and MTP, in a concentration-dependent manner, confirming that nonspecific binding was the cause of incomplete recovery in our PAMPA. Accordingly, the permeability of PPN, VER, and MTP also increased significantly with the addition of polysorbate 80 in a concentration-dependent manner. This indicates that the permeability of PPN, VER, and MTP measured in the absence of polysorbate 80 was underestimated because of nonspecific binding. Interestingly, polysorbate 80 caused a concentration-dependent decrease in the permeability of CLP. This seems to be unrelated to nonspecific binding as CLP showed complete recovery in the absence and presence of polysorbate 80 . It is postulated that this phenomenon is due to the binding of the compound to polysorbate 80 (or its micelle) although the reason why polysorbate 80 had a detrimental effect on the permeability of CLP alone remains to be elucidated. Nonetheless, CLP was classified correctly as a high permeability compound even in the presence of polysorbate 80 .

Polysorbate 80 has previously been used as a solubilizing agent for poorly soluble compounds in PAMPA assays and has no significant disruptive effect on phospholipid membranes in concentrations of $0.1-5 \%(\mathrm{w} / \mathrm{v}) .{ }^{10)}$ In our assay, the phospholipid membrane appeared to remain intact with up to $2 \%$ (vol) polysorbate 80, as demonstrated using calcein and Lucifer Yellow $\mathrm{CH}$ as hydrophilic fluorescent markers to assess membrane integrity.

We choose $0.3 \%$ (vol) polysorbate 80 as an additive to minimize nonspecific binding in our PAMPA assay because the increase in the recovery and permeability of PPN and VER as well as MTP reached a plateau at that concentration. The per- 
A

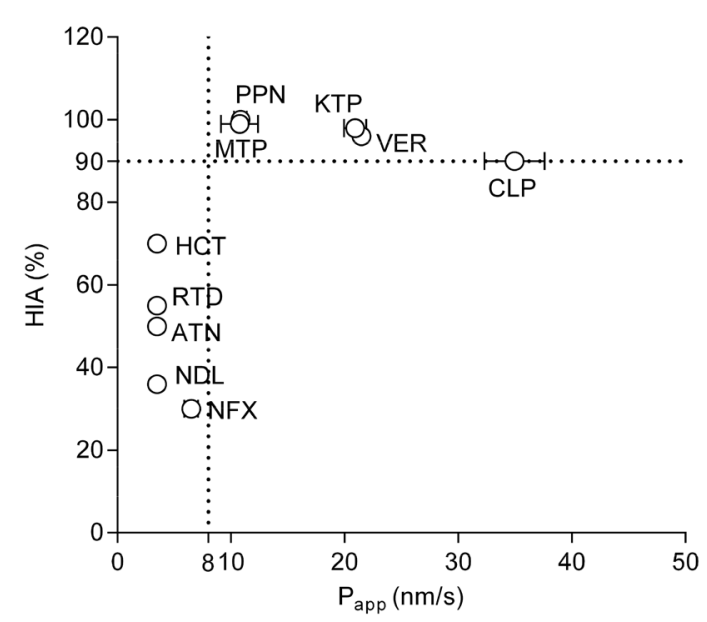

B

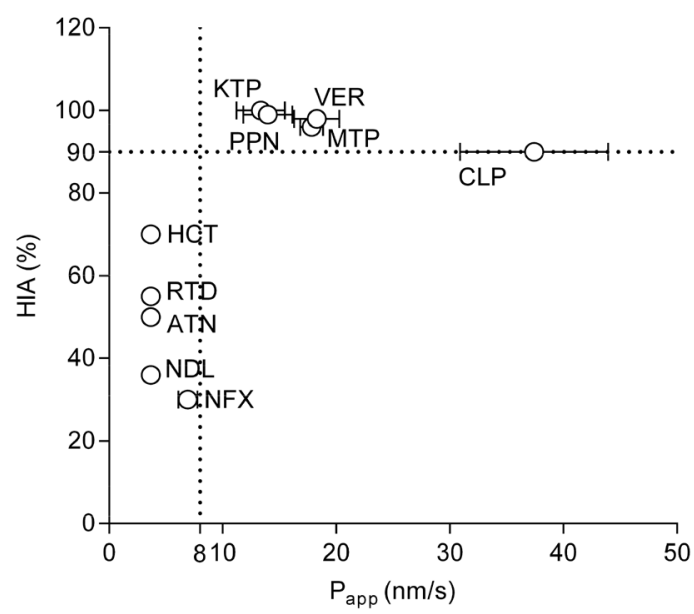

Fig. 5. Correlation between PAMPA Permeability Determined in the Presence of Polysorbate 80 in the Donor Compartments and HIA

$P_{\text {app }}$ values were determined in triplicate in cassette mode (A) as described in Fig. 1 or with each compound added separately to the donor compartments, i.e., in discrete mode (B).

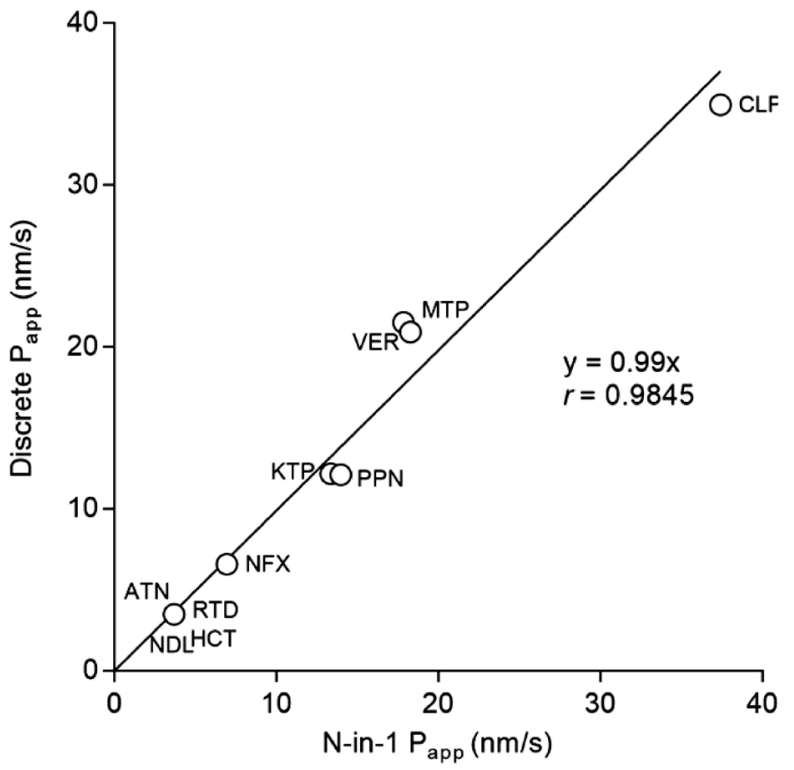

Fig. 6. Correlation of $P_{\text {app }}$ Values between Cassette and Discrete PAMPA Assays

The $P_{\text {app }}$ values were determined either in $n$-in- 1 or discrete mode as described in Fig. 4.

meability of all reference compounds measured could then be unequivocally classified into either a high or low permeability group using a $P_{\text {app }}$ value of $8 \mathrm{~nm} / \mathrm{s}$ as the cutoff. A strong linear relationship was observed between permeability measured in cassette and discrete modes, demonstrating that the permeability of each compound was not affected by the presence of the others in our cassette PAMPA.

This cassette PAMPA assay will be useful for permeability screening, especially in early stages of drug discovery when a number of diverse compounds, including highly lipophilic compounds, are produced and tested. At a minimum, this method will save resources by allowing a set of reference compounds with a wide range of lipophilicity to be run at once in the same well in PAMPA assays.
Acknowledgment This work was supported by the Basic Research Lab Program, National Research Foundation (Grant No. NRF-2014R1A4A1007304), Republic of Korea.

Conflict of Interest The authors declare no conflict of interest.

\section{REFERENCES}

1) Buckley ST, Fischer SM, Fricker G, Brandl M. In vitro models to evaluate the permeability of poorly soluble drug entities: challenges and perspectives. Eur. J. Pharm. Sci., 45, 235-250 (2012).

2) Volpe DA. Drug-permeability and transporter assays in Caco-2 and MDCK cell lines. Future Med. Chem., 3, 2063-2077 (2011).

3) Keserü GM, Makara GM. The influence of lead discovery strategies on the properties of drug candidates. Nat. Rev. Drug Discov., 8 , 203-212 (2009).

4) Tyrchan C, Blomberg N, Engkvist O, Kogej T, Muresan S. Physicochemical property profiles of marketed drugs, clinical candidates and bioactive compounds. Bioorg. Med. Chem. Lett., 19, 6943-6947 (2009).

5) Hann MM. Molecular obesity, potency and other addictions in drug discovery. MedChem Comm, 2, 349-355 (2011).

6) Carrara S, Reali V, Misiano P, Dondio G, Bigogno C. Evaluation of in vitro brain penetration: optimized PAMPA and MDCKII-MDR1 assay comparison. Int. J. Pharm., 345, 125-133 (2007).

7) Zhang J, Maloney J, Drexler DM, Cai X, Stewart J, Mayer C, Herbst J, Weller H, Shou WZ. Cassette incubation followed by bioanalysis using high-resolution MS for in vitro ADME screening assays. Bioanalysis, 4, 581-593 (2012).

8) Avdeef A, Artursson P, Neuhoff S, Lazorova L, Grasjo J, Tavelin S. Caco-2 permeability of weakly basic drugs predicted with the double-sink PAMPA p $K_{\mathrm{a}}$ (flux) method. Eur. J. Pharm. Sci., 24, 333-349 (2005).

9) Fortuna A, Alves G, Soares-Da-Silva P, Falcao A. Optimization of a parallel artificial membrane permeability assay for the fast and simultaneous prediction of human intestinal absorption and plasma protein binding of drug candidates: application to dibenz $[b, f]$ azepine-5-carboxamide derivatives. J. Pharm. Sci., 101, 530-540 (2012).

10) Liu H, Sabus C, Carter GT, Du C, Avdeef A, Tischler M. In vitro 
permeability of poorly aqueous soluble compounds using different solubilizers in the PAMPA assay with liquid chromatography/mass spectrometry detection. Pharm. Res., 20, 1820-1826 (2003).

11) Corti G, Maestrelli F, Cirri M, Zerrouk N, Mura P. Development and evaluation of an in vitro method for prediction of human drug absorption-II. Demonstration of the method suitability. Eur. J. Pharm. Sci., 27, 354-362 (2006).

12) Bibi HA, Di Cagno M, Holm R, Bauer-Brandl A. Permeapad for investigation of passive drug permeability: The effect of surfactants, co-solvents and simulated intestinal fluids (FaSSIF and FeSSIF). Int. J. Pharm., 493, 192-197 (2015).

13) Volpe DA. Application of method suitability for drug permeability classification. AAPS J., 12, 670-678 (2010).

14) Deferme S, Van Gelder J, Ingels F, Van Den Mooter G, De Buck S, Balzarini J, Naesens L, De Clercq E, Kinget R, Augustijns P. Intestinal absorption characteristics of the low solubility thiocarboxanilide UC-781. Int. J. Pharm., 234, 113-119 (2002). 